\title{
Efficient Symmetry Detection Using Local Affine Frames
}

\author{
Hugo Cornelius ${ }^{1}$, Michal Perdoch ${ }^{2}$, Jiří Matas $^{2}$, and Gareth Loy ${ }^{1}$ \\ ${ }^{1}$ CVAP, Royal Institute of Technology, Stockholm, Sweden \\ ${ }^{2}$ Center for Machine Perception, CTU in Prague, Czech Republic
}

\begin{abstract}
We present an efficient method for detecting planar bilateral symmetries under perspective projection. The method uses local affine frames (LAFs) constructed on maximally stable extremal regions or any other affine covariant regions detected in the image to dramatically improve the process of detecting symmetric objects under perspective distortion. In contrast to the previous work no Hough transform, is used. Instead, each symmetric pair of LAFs votes just once for a single axis of symmetry. The time complexity of the method is $n \log (n)$, where $n$ is the number of LAFs, allowing a near real-time performance. The proposed method is robust to background clutter and partial occlusion and is capable of detecting an arbitrary number of symmetries in the image.
\end{abstract}

\section{Introduction}

Symmetry is a visual and physical phenomenon, occurring both naturally and in manufactured artefacts and architecture. In this paper we will concentrate on bilateral symmetry where features are reflected about a central axis.

Human perception of symmetry has been well-studied. Psycho-physical evidence points to symmetry detection being a pre-attentive process [1] and playing a role in both signalling the presence of objects and directing visual attention [1]. It is not only humans who can detect symmetry. Bees, for example, have been shown to naturally choose to visit more symmetrical flowers [2].

Symmetry seldom occurs by accident. If two symmetric image regions are detected it is likely that these regions are related in the real world, and there is a good chance that they belong to the same object. Thus by detecting symmetry it is possible to start grouping or segmenting the image without prior knowledge of image content. As many objects exhibit some degree of symmetry, this provides a context-independent mechanism for hypothesising the presence, location and extent of such objects in a scene. Thus computer vision systems can benefit from symmetry detection in a manner not dissimilar to the psycho-physical systems discussed above.

This paper builds on recently published results 3/4 that illustrated the effectiveness of symmetry detection using local features. In this work we use local affine frames (LAFs) [5] constructed on affine covariant distinguished regions 6.77 to dramatically improve the process of detecting symmetric objects under 
perspective distortion, resulting in a simpler, more efficient and more robust approach. The use of LAFs makes it possible to hypothesise the axis of symmetry from a single symmetric pair of features enabling a very computationally efficient algorithm. Each matching pair of reflected LAFs is hypothesising one bilateral symmetry axis. The symmetry axis hypotheses are grouped into symmetric constellations about common symmetry foci, identifying both the dominant bilateral symmetries present as well as a set of symmetric features associated with each foci. Our method simultaneously evaluates symmetry across all locations, scales and orientations under affine and perspective projection. An important observation is, that any affine covariant detection and matching process which provides richer than just point-to-point correspondences can be used in a similar way.

The remainder of this paper is organised as follows, Section 2 reviews previous work on symmetry detection and gives an introduction to distinguished region detectors and local affine frames, Section 3 describes the method, Section 4 presents experimental results and discusses the performance of the method, and Section 5 presents our conclusions.

\section{Previous Work}

Symmetry detection is a well-studied field in computer vision, and comprise a significant body of work spanning over 30 years. Comparatively, distinguished regions and local affine frames are relatively recent developments in the computer vision field. Here we present a brief review of symmetry detection focusing on methods that use local features, and also provide an introduction to distinguished regions and local affine frames which provide the essential tool for streamlining the solution to the symmetry detection problem.

\subsection{Symmetry Detection}

Symmetry detection has found use in numerous applications ranging from facial image analysis [8] and vehicle detection [9], to 3D reconstruction [10] and visual attention [1112. Existing symmetry detection techniques can be broadly classified into global and local feature-based methods. Global approaches treat the entire image as a signal from which symmetric properties are inferred, whereas local feature-based methods use local features, edges, contours or boundary points, to reduce the problem to one of grouping symmetric sets of points or lines. The local-feature based approaches offers numerous advantages in particular their ability to more efficiently detect local symmetries in images that are not globally symmetric.

Mukherjee et al. [13] used local features called distinguished points detected on curves that are preserved under perspective or affine transformation. Affine semilocal invariants are used to match pairs and form symmetry hypotheses. The method was succesfully used to find symmetries on contours of simple objects. Recent methods 144 have increased robustness and effectiveness by exploiting 
detection and matching of richer features - distinguished regions. However they use the Hough transform to accumulate symmetry hypotheses which slows down the detection process. Moreover, the methods do not utilise the full extent of the information provided by affine matching techniques. In this work we shall show that using this additional information significantly simplifies and streamlines the symmetry detection process.

Tuytelaars et al. 14 presented a method for the detection of regular repetitions of planar patterns under perspective skew using a geometric framework. The approach detected all planar homologies and could thus find reflections about a point, periodicities, and mirror symmetries. The method built clusters of matching points using a cascaded Hough transform, and typically took around 10 minutes to process an image. In contrast the new method proposed herein provides a simpler and more efficient approach. We utilise local feature information to establish robust symmetric matches that directly vote for single symmetry hypotheses.

Recently Cornelius and Loy 4 proposed a method for grouping symmetric constellations of features and detecting symmetry in perspective. This approach was developed from 3 , which matched reflective feature pairs to detect symmetry in the image plane. The later method merged pairs of symmetrically matching features into feature quadruplets, so that each quadruplet defined a specific symmetry foci under perspective skew. The quadruplets were grouped into symmetric constellations of features about common symmetry foci, identifying the dominant symmetries present in the image plane. The disadvantage of this approach was the necessity to merge feature pairs into quadruplets. This was both time consuming and could easily result in spurious hypotheses.

Here we take the concept proposed by [4] and improve this by removing the need to form feature quadruplets, and thus solve symmetry detection under perspective in a cleaner and more efficient manner more concordant with the simple approach used to detect symmetry in the image plane 3 . That is, we show how to derive a unique symmetry hypothesis from a single pair of symmetric features, with the added challenge of an unknown perspective distortion.

\subsection{Distinguished Regions and Local Affine Frames}

The first step of the proposed algorithm is detection of distinguished regions followed by construction and description of local affine frames.

Distinguished regions are subsets of image pixels with some distinguishing property that allows their repeated detection over a range of image deformations, e.g. affine 11 transformations and photometric changes. Several affine covariant region detectors were presented and compared recently in [15]. Although any affine covariant region detector can be used, we have focused on only two kinds of regions in our work - Maximally Stable Extremal Regions (MSERs) 6 and Hessian-Affine regions [7. MSERs (see Fig. 1) are invariant to photometric

${ }^{1}$ In the framework described in this work we assume a locally planar scene under perspective projection. 


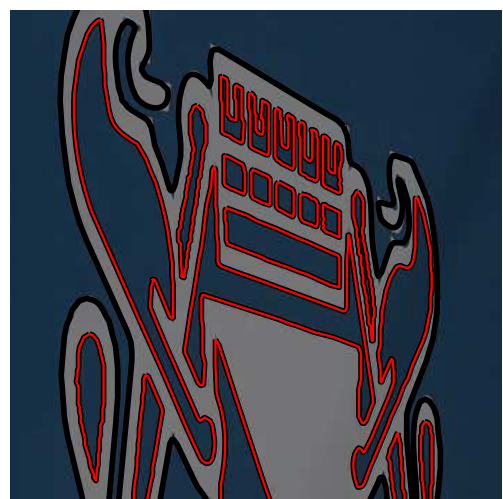

a)

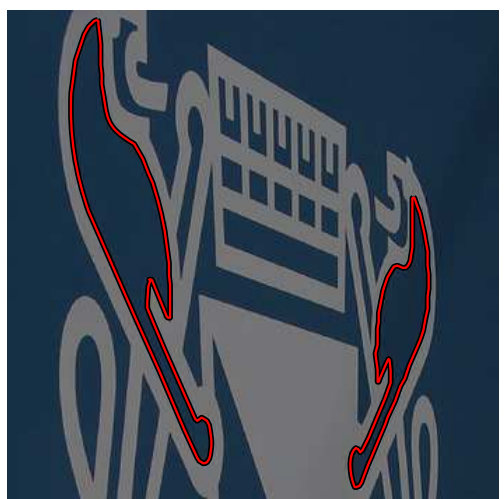

b)

Fig. 1. Distinguished Regions detection: a) detected maximally stable extremal regions, b) example of a reflected pair of MSERs

changes and covariant with all continuous transformations. The MSER detector generates simple polygons - contours that encompass interior pixels of stable regions. The Hessian-Affine detector first localises points in space and scale at local maxima of the Hessian and Laplacian-of-Gaussians. Affine covariant regions are then computed in an iterative affine adaptation process based on the second moment matrix of image gradients [16].

To resolve the affine transformations between distinguished regions in a single image a local affine frames approach [5] is exploited. Local affine frames (LAFs) are local coordinate systems constructed on distinguished regions covariantly with affine image transformations. LAFs provide an affine and photometric normalisation of local image patches into a canonical form. Geometrically and photometrically normalised patches can then be directly compared eliminating the need for invariants. The construction of the local affine frame resolves six free parameters of a two-dimensional affine normalisation. Two major groups of LAF constructions, based on shape and local image intensities were proposed in [5]. The shape based LAFs are formed by combining affine covariant measures, e.g. area, center of gravity and second moment matrix of the distinguished region, bi-tangent points, curvature extrema, point of inflections and other.

Intensity based LAF construction are computed from a center of gravity, covariance matrix and dominant gradient orientation (similar as in [17]) in the following way: First, a small image patch around the distinguished region is normalised using its centre of gravity and second moment matrix. Then a histogram of local gradient orientations is computed and dominant orientations are selected as local maxima of histogram bins. Each dominant orientation is used to fix the remaining parameter of an affine normalisation. In this work, the intensity based LAF construction is used with Hessian-Affine regions and shape based constructions with MSERs. 


\section{Detecting Bilateral Symmetry Using Local Affine Frames}

As in 3] and 4, the method for detecting planar bilateral symmetry under perspective skew presented here is based on pairwise matching of local features within a single image. The features used here are local affine frames (LAFs). To allow matching of bilaterally symmetric LAFs, a mirrored version of each frame and its descriptor from the previous step is computed. The mirroring depends only on the type of the local affine frame construction. The local affine frame construction, assumes a right-handed coordinate system when ordering the points and forming the frame. When constructing the mirrored frame, a left-handed coordinate system is used e.g. if a frame is constructed from the centroid and two points on the concavity - entry and exit point - the points on the concavity are swapped as the ordering on the contour of distinguished region is changed from clockwise to anti-clockwise. Other LAF constructions are mirrored in a similar manner without any knowledge about the axis of symmetry. Examples of LAFs and their mirrored versions are shown in Fig. 2.

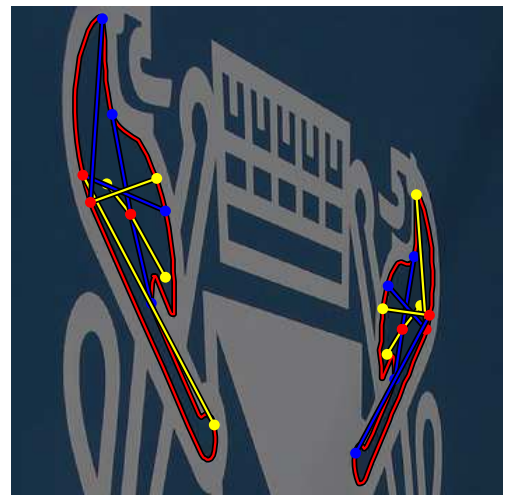

a)

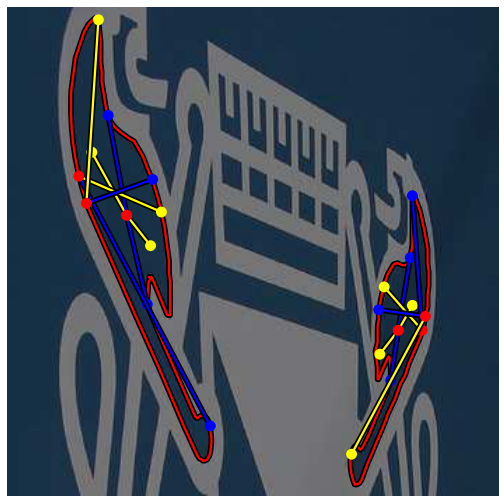

b)

Fig. 2. LAF constructions: a) Some of the local affine frames constructed on a maximally stable extremal region, b) Mirrored LAFs

In the next step LAFs are matched against their mirrored versions to find symmetric pairs. The naive approach would be to match every LAF to all mirrored ones and keep the $k$ best matches for every frame. Such a matching scheme has time complexity $O\left(n^{2}\right)$, where $n$ is the number of frames detected in the image. To avoid the $O\left(n^{2}\right)$ time complexity a decision tree is used as proposed in [18. The decision tree is trained offline on a set of LAFs computed from training image:2. In the matching phase frames are first entered into the tree in time proportional to $O(n \log (n))$ and then for each mirrored local affine frame a leaf

${ }^{2}$ A single random wide-baseline stereo pair of image was used to train the decision tree for all experiments. 


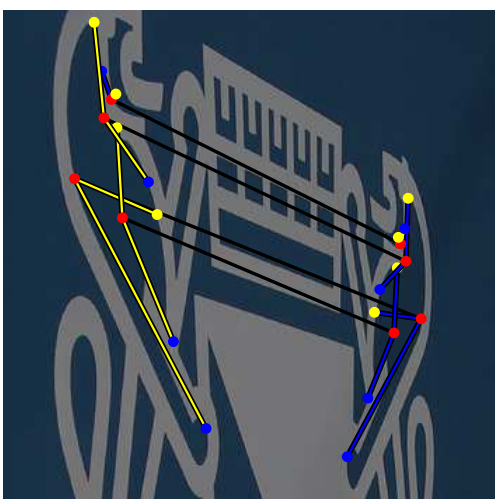

a)

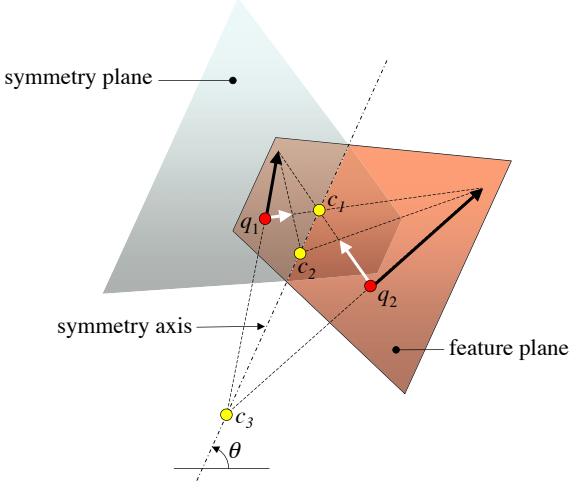

b)

Fig. 3. a) Some matching LAF pairs. Each pair can be used to form a hypothesis. b) A reflective pair of LAFs $\left(q_{1}, q_{2}\right)$ and the axis of symmetry around which the pair is symmetric. The intersections of the three lines defined by the three points in the LAFs all lie on the axis of symmetry $\left(c_{1}, c_{2}, c_{3}\right)$.

with similar frames is located in the tree in $O(\log (n))$. Candidates from a leaf are sorted using correlation of normalised patches and the $k$ best LAFs are returned. Throughout our experiments $k=3$ was used. The total time complexity of this matching scheme is $O(n \log (n))$ which in practise means that it is possible to achieve real-time performance.

When pairs of reflected frames have been obtained, an axis of symmetry is calculated for all pairs where possible. A LAF is defined by three points and three lines can be obtained by connecting these points. For a symmetric pair of frames, the three pairs of corresponding lines will intersect on the axis of symmetry. This means that the axis of symmetry can be estimated from a single pair of matching LAFs (see Fig. 3) if intersections $c_{1}, c_{2}$, and $c_{3}$ exist. Please note that this holds not only for symmetric patterns that lie in a plane, but also if the two halves of the symmetric pattern lie in different planes whose intersection coincides with the axis of symmetry. For pair of frames reflected about an axis of symmetry a set of necessary conditions has to hold:

1. The intersection, $c_{i}$, between a pair of lines defined by two points in the LAFs has to lie on the same side of both LAFs (see Fig. 33).

2. The three intersections $\left(c_{1}, c_{2}\right.$, and $\left.c_{3}\right)$ have to lie on a line (the axis of symmetry).

3. The axis of symmetry has to cross the line between the two LAFs.

Due to noise in the positions of the points in the LAFs, the second condition will in general not hold and small deviations from the line are allowed.

All matched pairs of frames for which these conditions hold, cast a vote for one axis of symmetry. Lines receiving many votes are identified as potential axes of symmetry and the pairs of frames that are consistent with the same 
axis of symmetry are grouped together. Even though just a small fraction of the feature pairs obtained in an image is correct, false symmetry axes will in general receive insignificant support. For a true symmetry axis the majority of the pairs consistent with it will be correct (inliers).

The lines joining pairs of features that are symmetric around the same axis of symmetry are parallel in 3D. This means that in an image, these lines will share a common vanishing point. For a planar bilaterally symmetric pattern, it is possible to estimate the vanishing point from the positions of the two features in a pair and the point where the axis of symmetry intersects the line between the features. The estimate will however be sensitive to small changes in the position of the axis of symmetry. Furthermore, if both halves of the symmetric pattern do not lie in the same plane but in two planes that intersect at the axis of symmetry, the intersections can not be used since they no longer correspond to the points that lie in the middle between the LAFs in 3D. It is however still possible to use the directions of the lines between the features and these will also be robust to small changes in the feature positions. We use the same approach as in [4] to estimate the vanishing point and reject the pairs that are not consistent with it. If $\psi_{i}$ is the orientation of the line between the two features in the $i$ :th pair of LAFs and $\theta$ is the direction perpendicular to the axis of symmetry, the following will hold:

$$
\tan \left(\psi_{i}-\theta\right)=\frac{h_{i}-p}{L}
$$

if $h_{i}$ is the point where the line between the two features in the pair intersects the axis of symmetry, $L$ is the distance from the vanishing point to the axis of symmetry and $p$ is the point on the axis of symmetry closest to the vanishing point. Since $\tan \left(\psi_{i}-\theta\right)$ is a linear function of $h_{i}, p$ and $L$ (that determine the vanishing point) can be estimated from two $\left(\psi_{i}, h_{i}\right)$-pairs, i.e. from two symmetric pairs of LAFs. RANSAC [19] is used to find a good estimate of $p$ and $L$ and to reject the pairs not consistent with the vanishing point. Since the number of inliers will typically be much larger than the number of outliers, a good estimate of the vanishing point will be found quickly.

The final output from the method is one or more symmetry axes and vanishing points together with the LAF and region pairs that are consistent with the symmetries.

\section{Experiments}

We have tested our symmetry detection method on a range of images of symmetric objects and scenes. In Fig. 4 some results of detected symmetries are shown. The examples contain a wide variety of objects both man-made, such as the buildings and the carpet, and natural (the butterflies and the face). Many images contain a considerable amount of background clutter (see e.g. Fig. 4(h), and some results with severe perspective skew are also shown (Fig. 4(a) and 4(b) . The butterflies are examples of symmetric patterns where the two halves of the patterns lie in different planes that intersect at the axis of symmetry. Especially in Fig. 4(i) the effect is quite clear. Symmetry detection on buildings is 


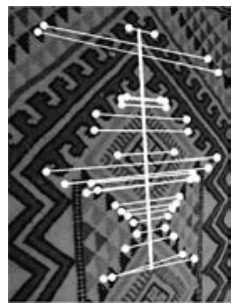

(a)

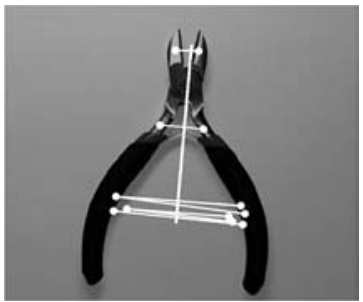

(e)

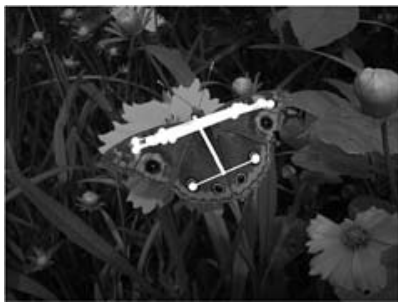

(h)

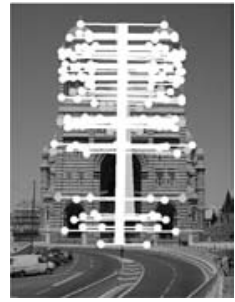

$(\mathrm{k})$

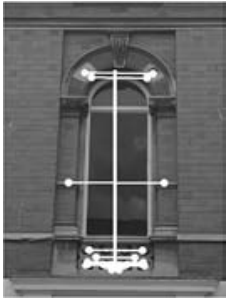

(l)

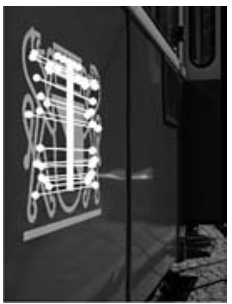

(b)

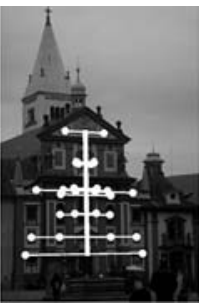

(c)

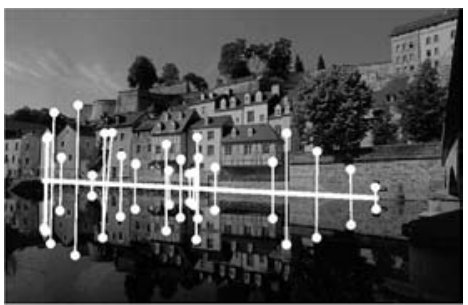

(f)

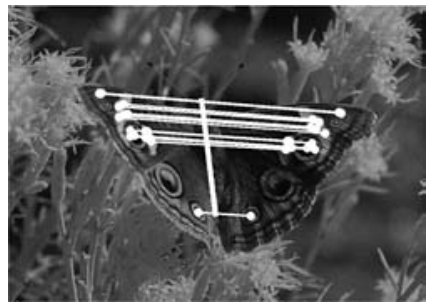

(i)

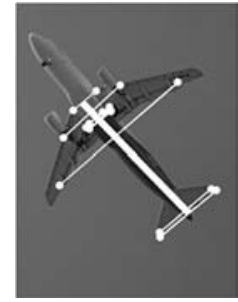

$(\mathrm{m})$

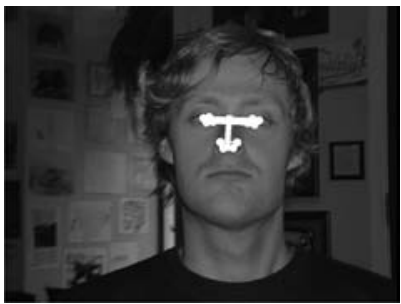

(d)

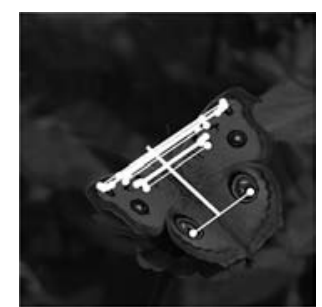

$(\mathrm{g})$

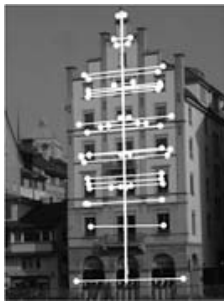

(j)

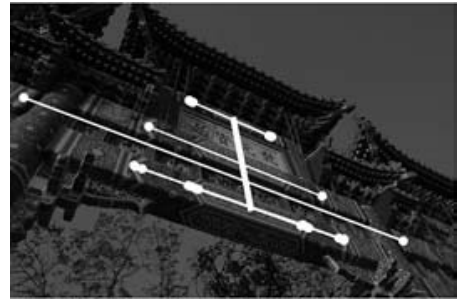

(n)

Fig. 4. Symmetry detected in perspective images, showing the reflective matches and axes of symmetry detected

complicated by the fact that a part of a building will be symmetric with respect to many other parts on the building around different symmetry axes. In the results presented in Fig. 4 our algorithm managed to identify the strongest and most global symmetry axis. All axes of symmetry with support higher than a threshold depending on the support for the strongest axis of symmetry detected in the image are shown. If that threshold would be lowered more symmetry axes would appear on the buildings. 
The results presented were generated using one of two different region detectors. Either MSERs [6] or Hessian-Affine regions [7]. During the experiments, we observed that the LAFs constructed on MSERs were more accurate than the ones constructed from the Hessian-Affine regions. This means that more accurate estimates of the axis of symmetry can be made from LAFs constructed on MSERs. Accuracy in the positions of the points in the LAFs is essential for the success of our algorithm. A small change in the position of a point sometimes leads to large changes in the estimated axis of symmetry due to the fact that the regions, and hence the LAFs, are usually much smaller than the distance between the regions in a pair.

Although the LAFs obtained from the Hessian-Affine regions were less accurate some good results can still be obtained. The results in the Figs. 4(a), 4(d) $4(\mathrm{f})$ 4(m) were obtained using the Hessian-Affine region detector.

A problem noted when testing the algorithm is that the symmetric surface often generates too few regions for the symmetry to be detected. Human faces are one example. In Fig. 4(d) it can be seen that only a few symmetric pairs of LAFs were detected on the eyes and the nose. To reduce this problem several region types could be used on the same image instead of just one at the time.

A natural extension to our current method would be to add a segmentation step at the end that would segment out the symmetric part of the image. Such a step would also verify the detected symmetry and could be used to reject incorrectly detected symmetries. A region growing algorithm like the one used in [14 could for example be used for this purpose.

\section{Conclusions}

In this paper we have presented an efficient method for detecting bilateral planar symmetry in images under perspective distortion. The method uses local affine frames constructed on maximally stable extremal regions or any other affine covariant regions. The complexity of the proposed algorithm is $n \log (n)$, where $n$ is the number of LAFs detected in the image allowing in practice a near realtime performance.

Hypotheses are generated from only one corresponding reflected pair of local affine frames and verified using the rest of the corresponding pairs. Hence our method is very robust to background clutter and able to discover multiple symmetries in the image in near real-time.

\section{Acknowledgements}

Authors were supported by STINT Foundation under project Dur IG2003-2 062, by the Czech Ministry of Education projects 1M0567 and by Czech Science Foundation project 102/07/1317. 


\section{References}

1. Dakin, S.C., Herbert, A.M.: The spatial region of integration for visual symmetry detection. Proc of the Royal Society London B. Bio. Sci. 265, 659-664 (1998)

2. Horridge, G.A.: The honeybee (apis mellifera) detect bilateral symmetry and discriminates its axis. J. Insect Physiol. 42, 755-764 (1996)

3. Loy, G., Eklundh, J.O.: Detecting symmetry and symmetric constellations of features. In: Leonardis, A., Bischof, H., Pinz, A. (eds.) ECCV 2006. LNCS, vol. 3952, Springer, Heidelberg (2006)

4. Cornelius, H., Loy, G.: Detecting bilateral symmetry in perspective. In: Proc of 5th Workshop on Perceptual Organisation in Computer Vision (POCV) (2006)

5. Obdrzalek, S., Matas, J.: Object recognition using local affine frames on distinguished regions. In: BMVC (2002)

6. Matas, J., Chum, O., Urban, M., Pajdla, T.: Robust wide baseline stereo from maximally stable extremal regions. In: BMVC. 3D and Video (2002)

7. Mikolajczyk, K., Schmid, C.: An affine invariant interest point detector. In: Heyden, A., Sparr, G., Nielsen, M., Johansen, P. (eds.) ECCV 2002. LNCS, vol. 2350, pp. 128-142. Springer, Heidelberg (2002)

8. Mitra, S., Liu, Y.: Local facial asymmetry for expression classification. In: CVPR (2004)

9. Zielke, T., Brauckmann, M., von Seelen, W.: Intensity and edge-based symmetry detection with an application to car-following. CVGIP 58(2), 177-190 (1993)

10. Hong, W., Yang, A.Y., Huang, K., Ma, Y.: On symmetry and multiple-view geometry: Structure, pose, and calibration from a single image. IJCV (2004)

11. Loy, G., Zelinsky, A.: Fast radial symmetry for detecting points of interest. PAMI 25(8), 959-973 (2003)

12. Sela, G., Levine, M.D.: Real-time attention for robotic vision. Real-Time Imaging 3, 173-194 (1997)

13. Mukherjee, D.P., Zisserman, A., Brady, J.M.: Shape from symmetry-detecting and exploiting symmetry in affine images. In: Philosophical Transactions of the Royal Society of London, Series A, vol. 351, pp. 77-106 (1995)

14. Tuytelaars, T., Turina, A., Gool, L.J.V.: Noncombinatorial detection of regular repetitions under perspective skew. PAMI 25(4), 418-432 (2003)

15. Mikolajczyk, K., Tuytelaars, T., Schmid, C., Zisserman, A., Matas, J., Schaffalitzky, F., Kadir, T., Gool, L.V.: A comparison of affine region detectors. IJCV (2006)

16. Lindeberg, T., Gårding, J.: Shape-adapted smoothing in estimation of 3-d cues from affine deformations of local 2-d brightness structure. ICV (1997)

17. Lowe, D.G.: Distinctive image features from scale-invariant keypoints. IJCV 60(2), 91-110 (2004)

18. Obdrzalek, S., Matas, J.: Sub-linear indexing for large scale object recognition. In: BMVC (2005)

19. Fischler, M., Bolles, R.: Random sample consensus: A paradigm for model fitting with applications to image analysis and automated cartography. Communications of the ACM 24(6), 381-395 (1981) 